\title{
A Rare Case of Systemic Lupus Erythematosus with Gastric Ulcer and Acute Pancreatitis: A Case Report and Literature Review
}

\author{
Vijay Gayamª, b, Amrendra Kumar Mandala, Mazin Khalid ${ }^{\mathrm{a}}$, Jaspreet Kaler ${ }^{\mathrm{a}}$, Shivani Thapa ${ }^{\mathrm{a}}$, \\ Pavani Garlapatia ${ }^{a}$ Arshpal Gilla, Ragin Alex ${ }^{\text {a }}$, Binav Shrestha ${ }^{\mathrm{a}}$
}

\begin{abstract}
Systemic lupus erythematosus (SLE) is a chronic inflammatory disease which can manifest in many different organ systems. Gastrointestinal (GI) involvement is common in SLE, but the symptoms are usually mild. More severe GI complications including acute pancreatitis and peptic ulcer bleeding are rare but represent a significant risk of morbidity and mortality. We present a case of a 25-year-old Hispanic female with a severe SLE flare. The initial presentation included symptoms of hematemesis and epigastric abdominal pain secondary to both gastric ulceration and acute pancreatitis, an atypical presentation of an SLE flare. The non-specific symptom of abdominal pain makes both acute pancreatitis and gastric ulcer disease a clinical challenge; however, clinicians need to have a high suspicion for these conditions co-existing at the same time due to higher mortality rates.
\end{abstract}

Keywords: Systemic lupus erythematosus; Acute pancreatitis; Gastric ulcer

\section{Introduction}

Systemic lupus erythematosus (SLE) is a chronic inflammatory condition that can affect virtually any organ. Gastrointestinal (GI) manifestations are common in SLE from $22-50 \%$ of patients experiencing GI symptoms, but they are usually mild; nevertheless, GI manifestations in SLE tend to dictate a higher mortality than SLE patients without GI complications $[1,2]$. In the case report below, we present a patient who presented to our hospital with hematemesis and epigastric pain, which was eventually diagnosed as a gastric ulcer and acute pancreatitis, an atypical presentation of an SLE flare.

Manuscript submitted May 15, 2018, accepted June 17, 2018

aDepartment of Medicine, Interfaith Medical Center, Brooklyn, NY, USA ${ }^{b}$ Corresponding Author: Vijay Gayam, Department of Medicine, Interfaith Medical Center, Brooklyn, NY 11238, USA.

Email:vgayam@interfaithmedical.com

doi: https://doi.org/10.14740/gr1048w

\section{Case Report}

A 25-year-old female, of Hispanic descent, with a history of SLE and class III lupus nephritis, presented to the emergency room with a 1-day history of hematemesis and epigastric pain. The patient denied any recent use of non-steroidal antiinflammatory drugs (NSAIDS), alcohol or anticoagulants. The patient was previously on tacrolimus and corticosteroids but had been non-compliant for 6 months due to a financial burden. She did not complain of any melena, weight loss, fever or joint pain.

Admission vitals revealed an afebrile patient with tachycardia of 127 beats per minute, a respiratory rate of 20 breaths per minute and a blood pressure of $90 / 58 \mathrm{~mm} \mathrm{Hg}$. Her physical examination was remarkable for mild epigastric tenderness, no guarding, no rigidity, small petechial rashes, notable pallor, decreased breath sounds bilaterally and pleuritic chest pain on the right side. There were no signs of icterus, ascites or organomegaly.

Initial investigations (Table 1) were significant for hemoglobin of $5.7 \mathrm{~g} / \mathrm{dL}$ (reference range: $12-15 \mathrm{~g} / \mathrm{dL}$ ) and a platelet count of $18,000 / \mathrm{mm}^{3}$ (reference range: $150,000-400,000$ / $\mathrm{mm}^{3}$ ). The patient's international normalized ratio (INR) was within the reference range. Leukocyte count was normal and blood smear showed no schistocytes. The chemistry panel revealed a sodium of $129 \mathrm{mEq} / \mathrm{L}$ (reference range: $136-144$ $\mathrm{mEq} / \mathrm{L}$ ), a potassium of $5.0 \mathrm{mEq} / \mathrm{L}$ (reference range: $3.6-5.1$ $\mathrm{mEq} / \mathrm{L}$ ) and a bicarbonate of $12 \mathrm{mEq} / \mathrm{L}$ (reference range: 22 $32 \mathrm{mEq} / \mathrm{L}$ ). Other significant laboratory results showed a total protein of $4.1 \mathrm{~g} / \mathrm{dL}$ (reference range: $6.1-7.9 \mathrm{~g} / \mathrm{dL}$ ) and an albumin of $1.2 \mathrm{~g} / \mathrm{dL}$ (reference range: $3.5-5.8 \mathrm{~g} / \mathrm{dL}$ ). Liver enzymes were within reference range. Her serum lipase was 5,039 U/L (reference range: 22 - 51 U/L) and serum amylase was $979 \mathrm{U} / \mathrm{L}$ (reference range: 28 - $100 \mathrm{U} / \mathrm{L}$ ). An abdominal computed tomography (CT) scan showed diffuse peri-pancreatic stranding consistent with acute pancreatitis (Fig. 1). Ultrasonography showed no evidence of cholelithiasis. The patient denied any history of alcohol abuse. The patient's serum calcium was not elevated and her serum triglycerides were only mildly elevated at $348 \mathrm{mg} / \mathrm{dL}$ (reference range: $0-149 \mathrm{mg}$ / $\mathrm{dL}$ ). She was not on any medications at the time of her admission. Serum IgG4 levels were not elevated. She was started on intravenous pantoprazole infusion. 
Table 1. Significant Laboratory Data at Admission

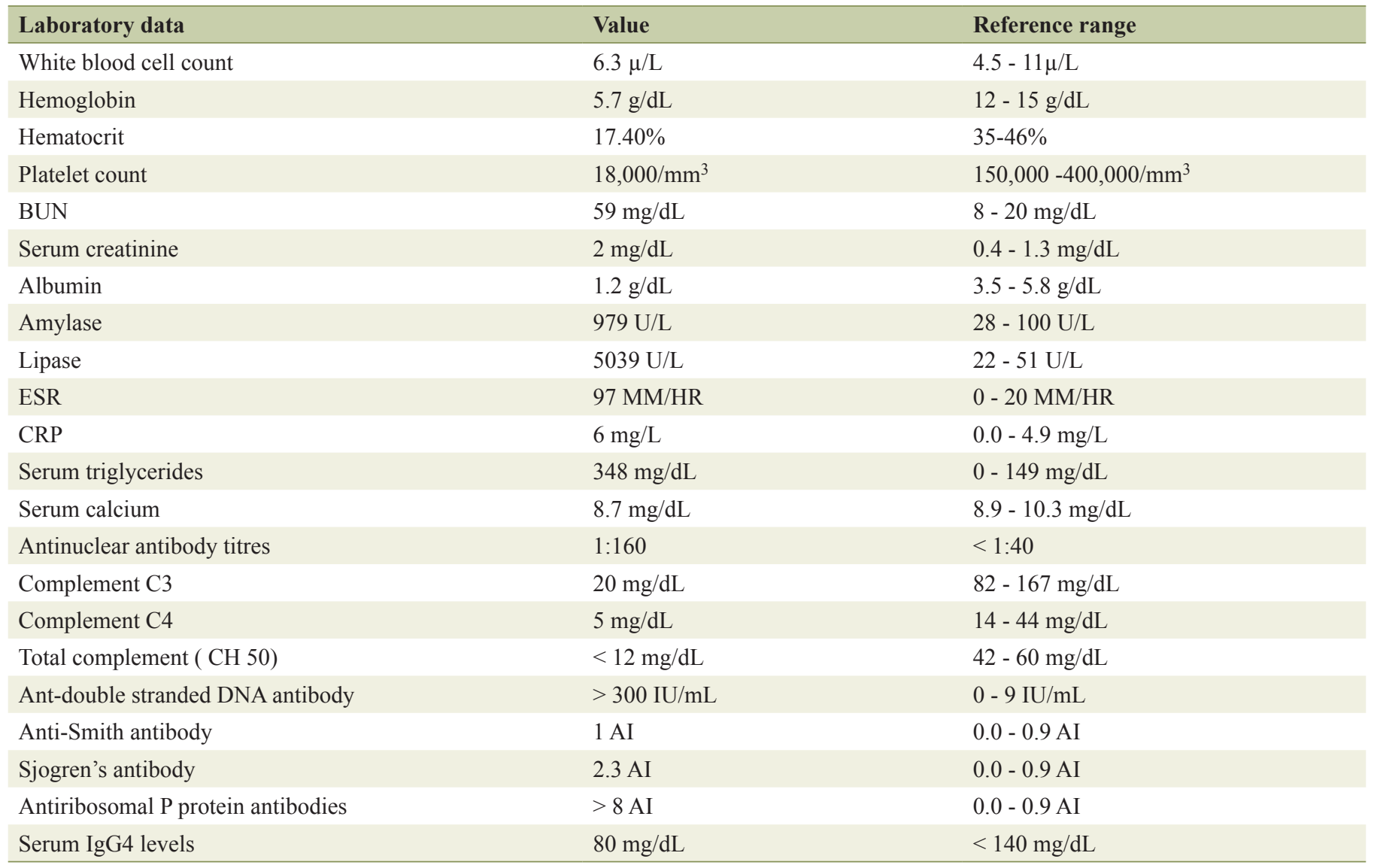

Esophagogastroduodenoscopy (EGD) and colonoscopy to look for the source of bleeding were performed after packed red blood cell and platelet transfusions were administered. The EGD revealed a $2 \times 2 \mathrm{~cm}$ ulcer with visible vessel (Forrest Classification: 2A) on the lesser curvature of the stomach, the ulcer with clipped and hemostasis was achieved (Fig. 2, 3). Gastric biopsy and stool antigen assay for Helicobacter pylori were negative. The colonoscopy was normal. Biopsy results of the stomach showed non-neoplastic gastric mucosa with chronic inflammation. (Fig. 4).

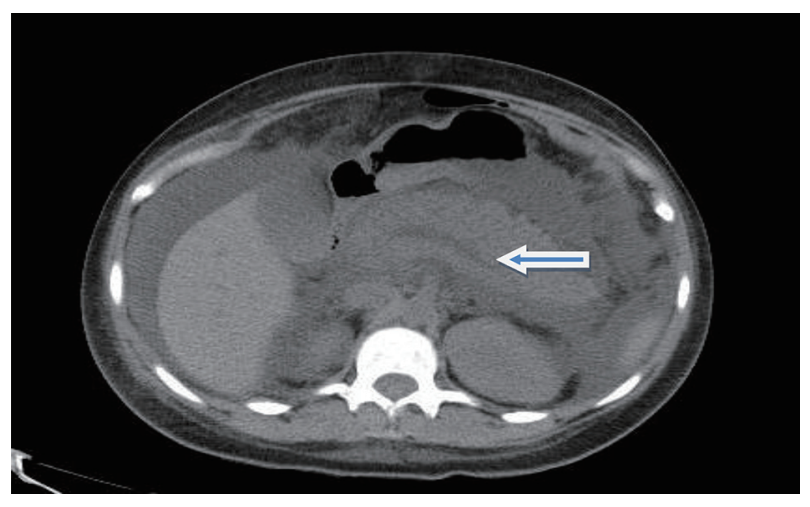

Figure 1. CT scan of the abdomen showing acute pancreatitis (arrow).
Immunology panel (Table 1) showed a decreased C3, C4, total complement, elevated antinuclear antibody titers, an elevated anti-dsDNA antibody, positive anti-Smith antibody, positive Sjogren antibody and positive anti-ribosomal $\mathrm{P}$ antibody. Cardiolipin antibodies, Scl-70 antibodies, C-ANCA and thyroid antibodies were all negative. Erythrocyte sedimentation rate (ESR) and C-reactive protein (CRP) were elevated.

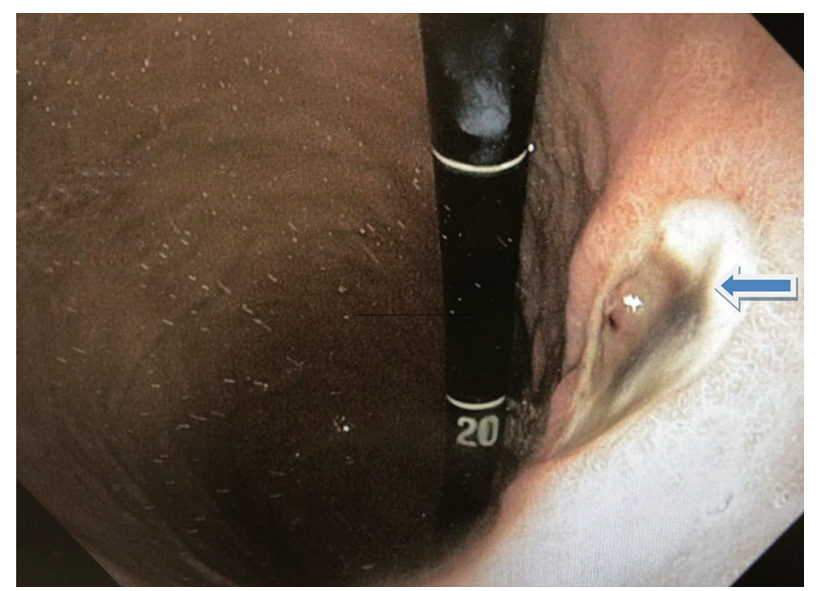

Figure 2. Gastric ulcer with the visible vessel in the lesser curvature (arrow). 


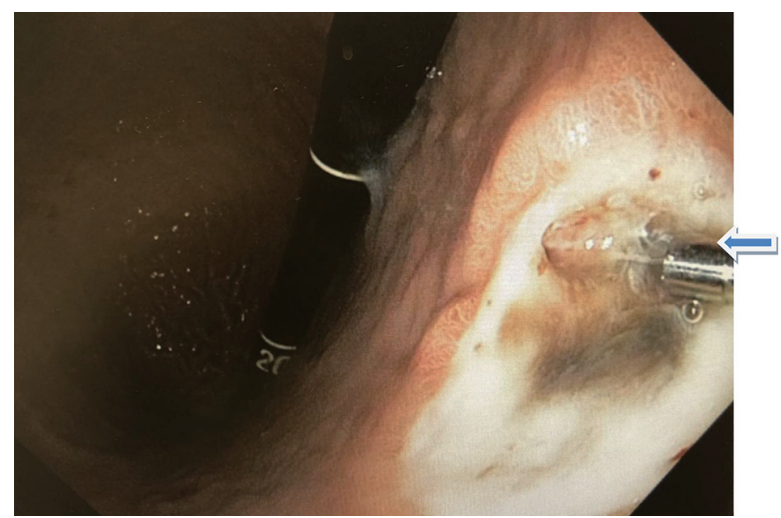

Figure 3. Gastric ulcer in the lesser curvature post hemostatic clipping (arrow).

Urinalysis showed hematuria and 5 - 15 leukocytes per high powered field. Intravenous methylprednisolone was initiated for the treatment of SLE flare.

Her hospital course was further complicated by other manifestations of SLE. The patient had acute kidney injury (AKI) with her serum creatinine rising to $4.1 \mathrm{mg} / \mathrm{dL}$ and serum blood urea nitrogen of $80 \mathrm{mg} / \mathrm{dL}$. Her clinical condition deteriorated and ultimately required hemodialysis. She developed acute respiratory failure with the chest CT scan (Fig. 5) showing a moderate right pleural effusion with multiple, small, ill-defined patchy opacities scattered throughout both lungs. All of this was consistent with cryptogenic complex organiz-

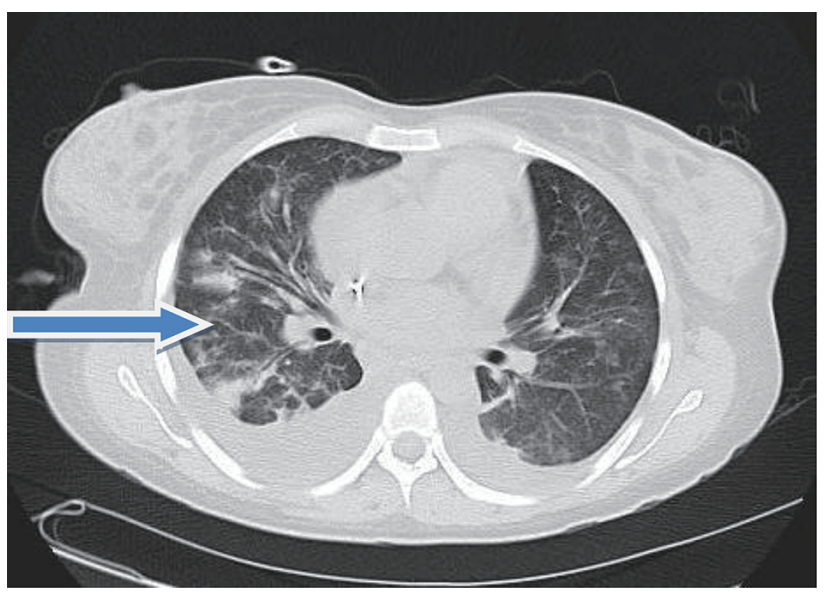

Figure 5. CT scan of the lung showing cryptogenic complex organizing pneumonia (arrow).

ing pneumonia. Sputum cultures grew vancomycin-resistant Staphylococcus aureus, which required treatment with daptomycin. The patient eventually recovered from the SLE flare and was discharged after 2 months of hospitalization on oral prednisolone, cyclophosphamide and pantoprazole.

\section{Discussion}

The patient in the case above presented with two simultane-

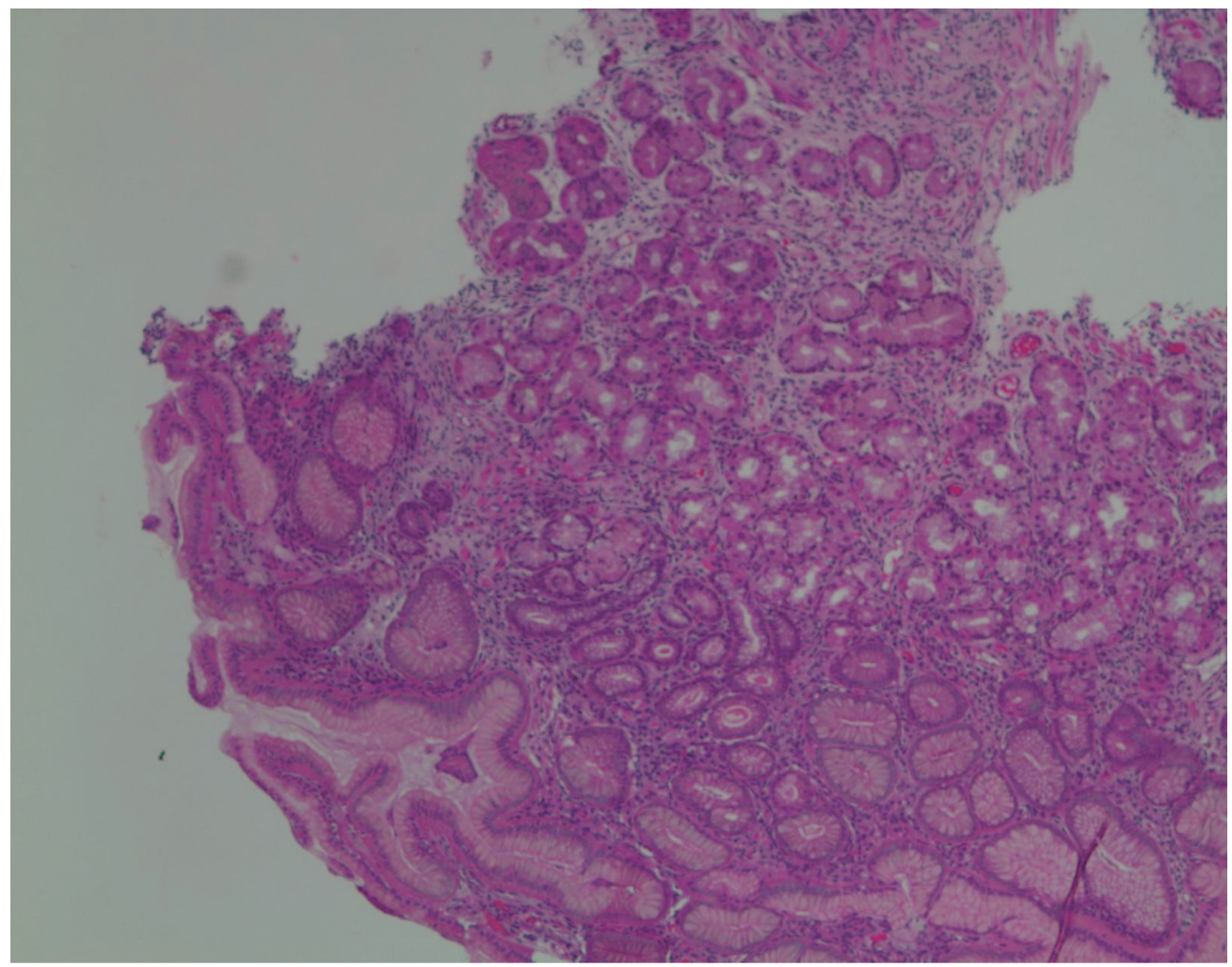

Figure 4. Histology (gastric mucosa) showing chronic inflammation. 
ous severe GI complications of SLE: peptic ulcer disease and acute pancreatitis. SLE flare was supported with an elevated ESR and anti-dsDNA antibody titers. In addition, there was a decrease in C3, C4, total complement and an elevated antinuclear antibody titers.

One of the major barriers to early recognition of acute pancreatitis is its non-specific findings. The most common symptom of acute pancreatitis in SLE patients is abdominal pain, which is seen in $88-93 \%$ of patients [3]. Unfortunately, abdominal pain is a very common finding seen in numerous conditions, including peptic ulcer disease, which was also comorbidity in this patient.

The diagnostic workup for SLE-related acute pancreatitis should include ruling out other etiologies for acute pancreatitis including gallstones, alcohol, hypertriglyceridemia, drugs, hypercalcemia and $\mathrm{IgG} 4$ disease. Since other etiologies were ruled out in our case, SLE flare was considered the reason for the simultaneous occurrence of peptic ulcer bleeding and acute pancreatitis $[1,5]$.

The reported incidence of acute pancreatitis associated with SLE varies from $0.85 \%$ to $4.2 \%[3,4]$. In a study by Nesher et al, 21 out of 73 patients with an SLE flare had acute pancreatitis [3]. Conversely, acute pancreatitis is a common cause of acute abdominal pain in SLE patients [5]. However, the mechanism of acute pancreatitis in SLE continues to remain unclear. Some of the possible mechanisms include autoimmune inflammation, anti-phospholipid syndrome causing thrombosis, and vasculitis [3]. There are no documented case reports of SLE patients presenting with acute pancreatitis and upper gastrointestinal (UGI) bleeding occurring simultaneously in the review of the literature. The likely mechanism could be explained by vasculitis due to SLE flare for both of the GI manifestations. Vasculitis leads to ischemia and infarction, progressing from superficial ulcerations to deep lesions [1, 5].

SLE patients may experience gastric ulcers for a variety of reasons, including iatrogenic and autoimmune. Although steroids are commonly used in SLE patients, steroids alone are not associated with an increased risk for peptic ulcer disease; instead, there is an asynergistic ulcerating effect on gastric tissue with concurrent NSAID use. SLE causing an additional ulcerogenic effect with NSAIDs and/or steroids is unknown [7]. Additionally, it is unknown if SLE has an increased risk of peptic ulcer disease distinguishable from NSAIDs and Helicobacter pylori infection $[2,8]$.

In the above case, we ruled out Helicobacter pylori and NSAIDS (the two most common causes of peptic ulcer disease) [9]. There was a history of steroid use 6 months prior to admission, but the lack of temporality and the absence of NSAID use in the history suggests that steroids are an unlikely cause of gastric ulcer bleeding in this patient. Furthermore, the improvement of the gastric ulcer after the initiation of steroid treatment indicates that it was related to an SLE flare and not because of previous steroid use. Therefore, SLE flare with vasculitis was the most reasonable explanation for a gastric ulcer in our patient $[1,5]$. Musaev et al revealed that SLE itself may cause gastritis [10], showing support that gastric ulcers may be primarily autoimmune in this SLE patient.

The review of the literature indicates that steroids significantly improve the outcome of acute pancreatitis in SLE [3,
6]. Contrary to the common practice that steroid use should be avoided in GI bleeding, an SLE flare and peptic ulcer bleeding actually respond well to corticosteroids, in conjunction with cyclophosphamide pulse therapy [5].

SLE patients with acute pancreatitis are more likely to have a severe presentation of SLE. A cohort study done by Yang et al identified that the SLE disease activity index (SLEDAI) is significantly higher in patients who incur acute pancreatitis as a manifestation [6].There is an increased number of organs involved and a higher mortality rate. The most accurate predictor of mortality in SLE-associated acute pancreatitis is the severity of pancreatitis [6]. Mortality of acute pancreatitis in SLE may be as high as $27 \%$ which emphasizes the urgency for early recognition and treatment. In a study of 35 SLE patients with 49 episodes of acute pancreatitis, the majority of cases were idiopathic and there was no evidence to suggest that the use of glucocorticoids or azathioprine were the culprit, which are commonly used drugs in SLE $[3,6]$.

During the hospital course, the patient developed acute kidney failure due to lupus nephritis flare requiring hemodialysis and cryptogenic organizing pneumonia (COP) with a secondary infection requiring antibiotics. We also ruled out mesenteric vasculitis (a rare and lethal manifestation of SLE during a flare) by the absence of thumbprinting or segmental bowel dilatation on plain radiographic imaging and absence of focal or diffuse bowel wall, mesenteric edema or engorgement of mesenteric vessels in abdominal CT. Another rare GI manifestation like SLE peritonitis was also looked for and was not found [11]. Imaging modalities like ultrasonography and abdominal CT were negative for ascites. Since the patient improved with steroids, cyclophosphamide and mechanical ventilation, this suggests that earlier administration of steroids and or immunosuppressive agents may significantly improve the outcomes of an SLE flare, preventing further complications.

\section{Conclusions}

Although simultaneous occurrences of acute pancreatitis and gastric ulcer bleeding are rare presentation for an SLE flare, clinicians must acknowledge the possibility, as it is associated with higher morbidity and mortality. Since non-specific abdominal pain is the chief complaint in both diseases and there is a high mortality rate, a prompt diagnosis is warranted. These patients should be treated with steroids and/or immunosuppressants to treat the underlying disease and to improve patient outcomes.

\section{Conflict of Interest}

None.

\section{Ethics Approval}

Our institution does not require ethical approval for reporting 
individual cases or case series.

\section{Informed Consent}

Written informed consent was obtained from the patient for anonymized patient information to be published in the article.

\section{References}

1. Xu D, Yang H, Lai CC, Li P, Zhang X, Yang XO, Zhang $\mathrm{FC}$, et al. Clinical analysis of systemic lupus erythematosus with gastrointestinal manifestations. Lupus. 2010;19(7):866-869.

2. Ebert EC, Hagspiel KD. Gastrointestinal and hepatic manifestations of systemic lupus erythematosus. J Clin Gastroenterol. 2011;45(5):436-441.

3. Nesher G, Breuer GS, Temprano K, Moore TL, Dahan D, Baer A, et al. Lupus-associated pancreatitis. Seminars in arthritis and rheumatism; 2006: Elsevier.

4. Makol A, Petri M. Pancreatitis in systemic lupus erythematosus: frequency and associated factors - a review of the Hopkins Lupus Cohort. J Rheumatol. 2010;37(2):341345 .

5. Hiraishi H, Konishi T, Ota S, Shimada T, Terano A, Sugimoto T. Massive gastrointestinal hemorrhage in systemic lupus erythematosus: successful treatment with corticosteroid pulse therapy. Am J Gastroenterol. 1999;94(11):3349-3353.

6. Yang Y, Ye Y, Liang L, Wu T, Zhan Z, Yang X, Xu H. Systemic-lupus-erythematosus-related acute pancreatitis: a cohort from South China. Clin Dev Immunol. 2012;2012:568564.

7. Medina F, Ayala A, Jara LJ, Becerra M, Miranda JM, Fraga A. Acute abdomen in systemic lupus erythematosus: the importance of early laparotomy. Am J Med. 1997;103(2):100-105.

8. Luo JC, Chang FY, Chen TS, Ng YY, Lin HC, Lu CL, Chen CY, et al. Gastric mucosal injury in systemic lupus erythematosus patients receiving pulse methylprednisolone therapy. Br J Clin Pharmacol. 2009;68(2):252-259.

9. Huang JQ, Sridhar S, Hunt RH. Role of Helicobacter pylori infection and non-steroidal anti-inflammatory drugs in peptic-ulcer disease: a meta-analysis. Lancet. 2002;359(9300):14-22.

10. Musaev SN, Novikova AV, Shershevskaia A, Klimanskaia EV, Aksenova IV. [The morphometric and immunohistochemical characteristics of the gastric and duodenal mucosa in systemic lupus erythematosus]. Biull Eksp Biol Med. 1991;111(2):203-206.

11. Ko SF, Lee TY, Cheng TT, Ng SH, Lai HM, Cheng YF, Tsai CC. CT findings at lupus mesenteric vasculitis. Acta Radiol. 1997;38(1):115-120. 